\title{
Solution Processing of Two-Dimensional Black Phosphorus
}

DOI:

10.1039/C6CC09658A

\section{Document Version}

Accepted author manuscript

Link to publication record in Manchester Research Explorer

\section{Citation for published version (APA):}

Lewis, E., Brent, J., Derby, B., Haigh, S., \& Lewis, D. (2017). Solution Processing of Two-Dimensional Black Phosphorus. Chemical Communications, 53. https://doi.org/10.1039/C6CC09658A

\section{Published in:}

Chemical Communications

\section{Citing this paper}

Please note that where the full-text provided on Manchester Research Explorer is the Author Accepted Manuscript or Proof version this may differ from the final Published version. If citing, it is advised that you check and use the publisher's definitive version.

\section{General rights}

Copyright and moral rights for the publications made accessible in the Research Explorer are retained by the authors and/or other copyright owners and it is a condition of accessing publications that users recognise and abide by the legal requirements associated with these rights.

\section{Takedown policy}

If you believe that this document breaches copyright please refer to the University of Manchester's Takedown Procedures [http://man.ac.uk/04Y6Bo] or contact uml.scholarlycommunications@manchester.ac.uk providing relevant details, so we can investigate your claim.

\section{OPEN ACCESS}




\section{Journal Name}

\section{ARTICLE}

\section{Solution Processing of Two-Dimensional Black Phosphorus}

Received 00th January 20xx, Accepted 00th January 20xx

DOI: $10.1039 / \times 0 \times x 00000 x$

www.rsc.org/

\begin{abstract}
Edward A. Lewis, ${ }^{a}{ }^{\dagger}$ Jack R. Brent, ${ }^{a}+$ Brian Derby, ${ }^{a}$ Sarah J. Haigh ${ }^{a}$ and David J. Lewis ${ }^{a} *$
Phosphorene, or two-dimensional (2D) black phosphorus (BP) was the first synthetic 2D elemental allotrope beyond graphene to be isolated and studied. It is useful due to its high p-type carrier mobility and direct band gap that is tunable in the range ca. $0.3-2 \mathrm{eV}$ thus bridging the energy gap between graphene and transition metal dichalcogenides such as molybdenum disulfide. Beyond the 'Scotch-Tape' method that was used to isolate the first samples of 2D BP for prototype studies, a range of potentially scalable solution processing techniques emerged later that can produce electronics grade material. This feature article focuses on such solution-process routes to 2D BP and highlights challenges in processing the material, mainly caused by its susceptibility to oxidation, as well as illuminating new avenues and opportunities in the area.
\end{abstract}

\section{Introduction}

Since the isolation of graphene over a decade ago, twodimensional (2D) materials have been the focus of a vast research effort, which shows no sign of decreasing in either intensity or output. ${ }^{1}$ Attention was initially directed toward the exploration of graphene, followed by layered materials 'beyond graphene', particularly functionalised carbon nanosheets, inorganic boron nitride and metal dichalcogenides. ${ }^{1-3}$ More recently, researchers have looked even more widely beyond even these materials to the study of more exotic 2D nitrides, ${ }^{4}$ carbides $^{5,6}$ and perovskites ${ }^{7,8}$ and novel homoatomic materials, silicene, ${ }^{9}$ germanene, ${ }^{10}$ stanene $^{11}$ and phosphorene. ${ }^{12,13}$ Of these, phosphorene, the monolayer 2D analogue of semiconducting black phosphorus (BP) herein referred to as $2 \mathrm{D} B P$, has attracted attention as a result its electronic properties and relative simplicity of production, compared to other group IV and V monolayers. In 2014 phosphorene became the first homoatomic 2D material to have been isolated since the discovery of graphene. ${ }^{14}$ As a result of its exceptionally high $p$-type carrier mobility $(>1000$ $\mathrm{cm}^{2} \mathrm{~V}^{-1} \mathrm{~s}^{-1}$ ), tuneable band gap and orientation-dependant properties the material is considered an exceptional candidate for use in high frequency electronics, sensing and energy storage. $^{15-17}$

Bridgman's original reports of the synthesis of orthorhombic bulk BP noted the new material's high density

\footnotetext{
${ }^{a}$ School of Materials, University of Manchester, United Kingdom M13 9PL. + Contributed equally

*to whom correspondence should be addressed: Dr. David J. Lewis MRSC, email: david.lewis-4@manchester.ac.uk.
}

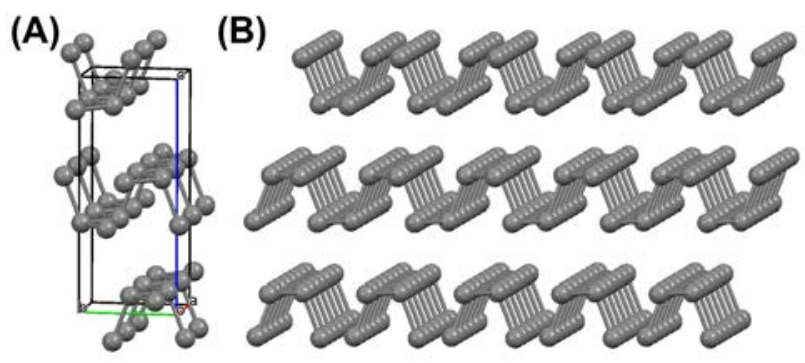

Figure. 1. The chemical structures of $B P$ and few-layer $B P$. (A) The orthorhombic unit cell of black phosphorus $(a=3.31 \AA, b=4.38 \AA \mathrm{A} c=10.50$ $\AA$, $\alpha=B=\gamma=90^{\circ}$; space group Bmab; Crystallography Open Database ID: $1010325)$ which generates a layer structure comprising corrugated lamellae of phosphorus atoms held together by weak interlayer forces. (B) Threelayer phosphorene. Figure reproduced from Brent et al. Chem. Commun. 2014, 50, 13338-13341, Published by The Royal Society of Chemistry.

and stability over the more common white and red phosphorus allotropes. ${ }^{18,19} \mathrm{BP}$ was found to consist of $A B$ stacked layers of $\mathrm{sp}^{3}$ hybridised phosphorus at an interlayer distance of 5.2-5.3 $\AA$ (Figure 1). ${ }^{20}$ Rather than a planar structure similar to graphite, BP layers are buckled, giving rise to anisotropic bond angles. Each $\mathrm{P}$ atom has three equidistant $(2.18 \AA ̊)$ nearest neighbours; two in-plane and one-out-ofplane, at bond angles of $99^{\circ}$ and $103^{\circ}$ respectively. This arrangement gives rise to armchair (AC) and zig-zag (ZZ) structures along perpendicular crystallographic axes, leading to highly anisotropic properties within the $a b$ plane. ${ }^{21-23}$

The Bridgman method relies on high pressure $(1.2 \mathrm{GPa})$ and moderately high temperature $\left(>200{ }^{\circ} \mathrm{C}\right)^{18,24}$ to transform white phosphorus into BP. Subsequent attempts to reduce the need for high pressure conditions focused on the use of molten bismuth ${ }^{25}$ and mercury fluxes, ${ }^{26}$ both of which are clearly problematic for large-scale use. It was not until 2007 that a kinetically controlled, low-pressure and non-toxic 
method was developed to obtain BP single crystals from red phosphorus. ${ }^{27}$ The method uses $\mathrm{Sn}$ and $\mathrm{Snl}_{4}$ mineralising agents in the presence of small amounts of gold to synthesise $\mathrm{BP}$ through the in situ formation of $\mathrm{Au}_{3} \mathrm{SnP}_{7}$. Subsequent work by the same group further reduced the need for high temperature and improved purity by introducing a vapour transport method. ${ }^{28}$ Nilges et al. have proposed that the reaction relies on the growth of specific reaction intermediates, which provide potential substrates for direct synthesis of ultrathin phosphorene. ${ }^{27}$ In a significant recent development, Akinwande et al. have reported a solutionprocessable route to black phosphorus via, sonication of red phosphorus powder. ${ }^{29}$ Ultrasonication has the potential to produce extremely high energy cavitation forces ${ }^{30}$ (vide infra) which, in this case, are sufficient to mimic the high temperatures and pressures used to produce BP in the gas phase. It was also determined that judicious choice of solvent and processing parameters can be used to produce violet phosphorus, another layered allotrope with potential in $2 \mathrm{D}$ electronics. ${ }^{31}$

Thinning any material to a few atomic layers induces significant deviations from bulk properties. Black phosphorus provides no exception, displaying band gap widening from approximately $0.3 \mathrm{eV}$ to up to $2 \mathrm{eV}$ when thinned to the monolayer limit, due to a reduction in the extent of interlayer hybridisation. ${ }^{14,}{ }^{32,33}$ Molybdenum disulfide $\left(\mathrm{MoS}_{2}\right)$ undergoes indirect/direct crossover in the bilayer to monolayer limit, ${ }^{34}$ with associated challenges for solution processing. In contrast, BP's band gap is direct at all thicknesses, implying that precise control over layer number may not be necessary for some applications. BP devices display carrier mobilities which vastly exceed those typical of $\mathrm{MoS}_{2}$ transistors. ${ }^{14,35-37}$ Few-layer BP therefore bridges the current gap between graphene and the 'traditional' 2D semiconductors by providing the high carrier mobility necessary for high-frequency electronics ${ }^{38}$ while maintaining an appreciable ON/OFF ratio. Several groups have

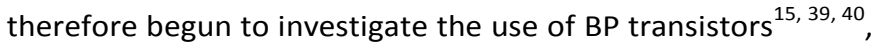
logic devices ${ }^{14}$ and memory storage ${ }^{41}$. Other potential applications include the use of 2D BP as an anode material in rechargeable batteries, ${ }^{17,42,43}$ and in the sensing of gas ${ }^{44}$ and biomolecules ${ }^{45}$ and in thermoelectric devices, ${ }^{21,46}$. The latter relies on the orthogonal preferred directions of electrical and thermal conductivity which arise from the anisotropic structure atomic structure of BP in the $a b$ plane. $^{47}$

The last two years of intensive investigation have determined that 2D BP possesses the requisite physical and electronic properties to have a great impact on green energy and next-generation electronics research. Several of the nanoscale devices which have already been produced have already surpassed the performance of conventional 2D semiconductors despite the infancy of phosphorene research. Groups have also developed several routes to nanoscale black phosphorus and begun to elucidate its chemistry, stability and potential for functionalisation. ${ }^{45,48-50}$

\section{Synthetic routes to 2D black phosphorus}

\subsection{Micromechanical exfoliation}

Micromechanical exfoliation refers to a top-down approach where an adhesive surface is used to pull bulk layered crystals apart to produce monolayer of few-layer materials. Also referred to as 'the Scotch-tape method', micromechanical exfoliation has been used to produce a wide range of $2 \mathrm{D}$ materials including graphene, ${ }^{51,52} \mathrm{~h}-\mathrm{BN},{ }^{53} \mathrm{MoS}_{2}{ }^{54,55} \mathrm{WSe}_{2}{ }^{56,57}$ $\mathrm{TaSe}_{2}{ }^{56} \mathrm{NbSe} 2{ }^{58} \mathrm{GaS}^{59}$ and $\mathrm{Bi}_{2} \mathrm{Te}_{3}{ }^{60}$ It is possible to isolate high quality crystals, which are relatively clean, monocrystalline, and of well-defined thickness. This method exploits the very low cleavage energy of the basal plane in the parent crystal structure of the 2D material and is well known in mineralogical circles as perfect pinacoidal cleavage of the bulk crystal. Micromechanically exfoliated flakes are commonly used for fundamental investigations into the physical properties and potential device applications of 2D materials. ${ }^{61}$

Micromechanical exfoliation was the first technique used to produce 2D crystals from bulk layered solids, ${ }^{62}$ and the first isolation of monolayer black phosphorus (BP) proved no exception. ${ }^{14,15}$ Using such an approach it is possible to produce monolayer single crystals with dimensions greater than a micron. For BP exfoliation it has been found that incorporating the dry transfer method, where material in exfoliated directly onto a viscoelastic stamp, ${ }^{63}$ can give higher yields of thin flakes. $^{64}$

Despite the high-quality of the crystals produced, the micromechanical exfoliation approach has a number of drawbacks. The approach is labour intensive, requiring hours of a skilled scientist's time. Furthermore, it is intrinsically unscalable. To fabricate a device, an individual thin flake must be identified by optical microscopy and then be isolated and transferred onto a new substrate. ${ }^{65-68}$ Sheets are produced and processed on a flake-by-flake basis, with human skill required at every step, and potentially introducing a range of random errors from subjective judgements. A further disadvantage of micromechanical cleavage is that the use of adhesive tape and polymer resists for exfoliation and transfer steps respectively often results in residual organic contamination. ${ }^{69}$ For black phosphorus there are additional challenges associated with its susceptibility to oxidation (vide infra).

\subsection{Liquid phase exfoliation in organic solvents}

While mechanical exfoliation produces nanosheets of extremely high quality, its usefulness is limited to laboratory scale investigations into the fundamental properties of 2D materials and the fabrication of proof-of-principle and prototype devices. Simple, scalable, and low-cost approaches are required if 2D materials are to find widespread applications. Liquid phase exfoliation (LPE) is a potentially scalable process where ultrasonic irradiation is used to exfoliate layered materials and produce colloidal dispersions of nanosheets in solution. In 2008 Coleman demonstrated the use of LPE to convert graphite flakes to graphene. ${ }^{70}$ The approach has since been successfully applied to a wide range of layered materials. ${ }^{71-75}$ We have been interested in using LPE linked to chemical vapour deposition (CVD) to produce a range 
of doped $^{76,77}$ and exotic 2D materials ${ }^{78}$ as well as using LPE to produce isoelectronic IV-VI BP analogues such as SnS. ${ }^{74}$ The colloids produced by LPE are compatible with established and commercially viable solution processing technologies such as inkjet printing and spin coating, offering simple routes by which materials and devices containing $2 \mathrm{D}$ materials could be manufactured. ${ }^{79}$ Due to these advantages we predict that LPE nanosheets will be the first form of $2 D$ materials to find widespread commercial applications.

When ultrasound (typically $20-50 \mathrm{kHz}$ ) is transmitted through a liquid the sound waves create alternating cycles of high and low pressure. In low pressure regions small voids are created which then collapse under compression, a process known as cavitation which results in the localised generation of high
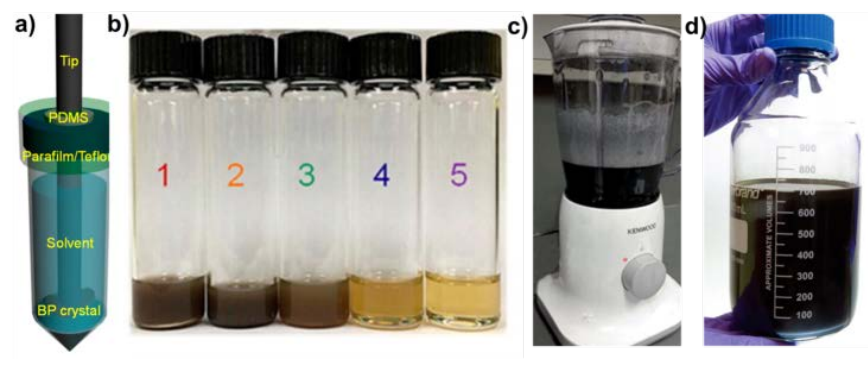

Figure 2. (a) Schematic demonstrating liquid phase exfoliation by probe sonication, (b) a series of LPE BP suspensions prepared by probe sonication in NMP and subjected to different centrifugation speeds (from left to right (1) as prepared, (2) $500 \mathrm{rpm}$, (3) 5000 rpm, (4) $10000 \mathrm{rpm}$, and (5) $15000 \mathrm{rpm}$ ). (c) Turbulent shear exfoliation of graphene using a kitchen blender. (d) BP suspension in NMP prepared by a combination of shear exfoliation and sonication. (a-b) adapted with permission from Kang et al. ACS Nano 2015, 9 (4), 3596-3604. Copyright 2016 American Chemical Society. (c) Reproduced from Varrla et al. Nanoscale, 2014, 6, 11810-11819, with permission from The Royal Society of Chemistry. (d) Adapted with permission from Woomer et al. ACS Nano 2015, 9 (9), 8869-8884 Copyright 2016 American Chemical Society.

temperatures and pressures. ${ }^{80}$ Ultrasonication of liquids can be performed using bath or probe type configurations; both experimental set-ups have been used to exfoliate BP successfully. ${ }^{81,82}$ In bath experiments the position of the sample in the bath can significantly affect the intensity of sonication, compromising reproducibility. ${ }^{83,} 84$ Ultrasonic probes are directly immersed into the solution to be sonicated (Fig. 1a), the power provided is typically higher than an ultrasonic bath, meaning shorter sonication times are required. ${ }^{84} \mathrm{~A}$ recent paper by Coleman and co-workers has highlighted best practices in the production of nanosheets by LPE. $^{85}$

LPE to produce 2D BP from bulk BP was first reported by our group in 2014. Bath sonication of BP in N-Methyl-2pyrrolidone (NMP) for 24 hours produced suspensions of nanosheets which were typically 3-5 layers thick. ${ }^{81}$ This work was rapidly followed by a flurry of studies into the LPE of BP in which altered experimental parameters were investigated in an effort to control sheet dimensions and yields. ${ }^{49,82,86,87}$ The solvent system used is a critical and widely investigated parameter in LPE. $^{82}$ Coleman et. al. performed liquid phase exfoliation of BP in N-cyclohyexyl-2-pyrrolidone (CHP). ${ }^{49}$ Hersam et al. compared exfoliation of BP in NMP, Acetone, Chloroform, Hexane, propan-2-ol (IPA), and dimethylformamide (DMF), concluding that NMP was the best of these solvents for producing ultrathin BP. ${ }^{82}$ Another study, assessing the suitability of 10 different solvents for BP exfoliation looked at maximising the concentration of colloidal material in suspension. It was found that the concentration of BP suspensions produced followed the order formamide > dimethyl sulfoxide (DMSO) > DMF, NMP, IPA > ethanol or methanol > acetone, tetrahydrofuran (THF), water. Interestingly, these results show a positive correlation between the solvent's surface tension and nanosheet concentration. $^{82,88}$ In another study 18 solvents were surveyed and benzonitrile was identified as optimal, based upon its ability to yield the highest concentration $\left(0.11 \mathrm{mg} \mathrm{mL}^{-1}\right) \mathrm{BP}$ suspensions. ${ }^{87}$ Perhaps the highest concentrations of BP dispersions produced to-date were achieved by LPE in ionic liquids with concentrations up to $0.95 \mathrm{mg} \mathrm{mL}^{-1}$ reported. ${ }^{89}$ It is worth noting that the concentration of suspensions produced is a relatively limited figure of merit to select solvents. The success of an exfoliation procedure will depend on a number of additional factors many of which are application specific. The thickness, lateral dimensions, size dispersity, crystal quality and stability of the nanosheets produced must all be considered, as must the compatibility of the chosen solvent with envisaged manufacturing processes and applications. Furthermore, both the cost and toxicity of the solvent are important factors to consider.
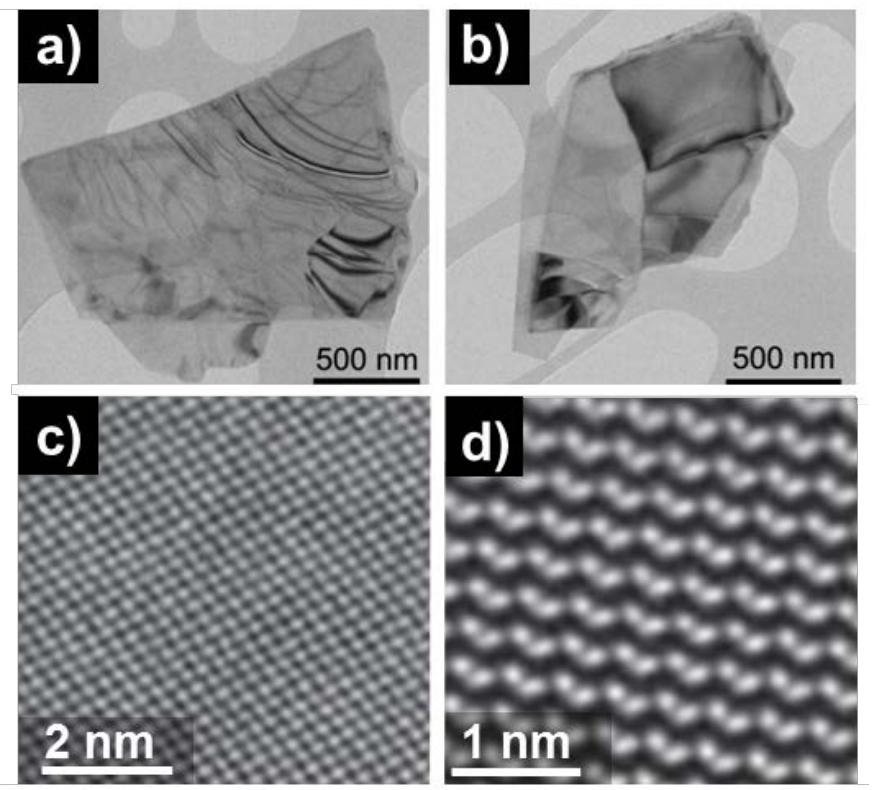

Figure 3. (Scanning) Transmission electron microscopy of BP flakes. (a-b) Low magnification TEM images of BP flakes exfoliated in CHP. Atomic resolution Adapted from Hanlon et al Nat. Comm. 2015, 6, 8563.

Given the thickness dependent properties of BP it is desirable to be able to quickly assess the thickness of the flakes within a 
BP suspension. Woomer et al. have demonstrated that optical absorption measurements can yield quantitative data on BP thickness (Figure 4). ${ }^{87}$ Their method relies on the assumption that absorption coefficients near the absorption edge remaining unchanged for any thickness between bulk and monolayer, allowing the effective band gap of a suspension (arising from a range of different sized flakes) to be estimated. ${ }^{87}$ The use of optical methods to determine the size, thickness, and concentration of LPE suspensions has also been demonstrated for $\mathrm{MoS}_{2}$ and $\mathrm{WS}_{2}{ }^{90}{ }^{90}$ We believe that widespread development and adoption of optical methods for size and thickness determination will result in a significant acceleration in the optimisation of LPE by reducing the need for time consuming statistical analysis by electron and scanning probe microscopies. Typical TEM images of flakes from LPE are shown in Figure 3.

Whilst the goal of many LPE studies is to produce thin nanosheets with large lateral dimensions a number of studies have looked at producing ultrathin flakes with very small lateral dimensions, described as quantum dots (QDs). ${ }^{41,92-94}$ Zhang et al. were able to produce BP QDs with lateral dimensions of $4.9 \mathrm{~nm}$ and thicknesses of $1.9 \mathrm{~nm}$ by sonication in NMP. ${ }^{41}$

The purity of solvents used may be important, in some reports considerable efforts are made to ensure that the solvents are anhydrous and oxygen free. ${ }^{82}$ The elimination of water and oxygen is typically motivated by the ease with which BP oxidises, this is discussed in depth is section 3. However, the presence of water has been shown the affect the exfoliation of BP in DMSO in other ways. The molar ratio of water to DMSO was systematically altered, with "dry" $\left(\mathrm{BP} / \mathrm{H}_{2} \mathrm{O}\right.$ $\geq 15)$, "intermediate" (14-1.5), and "wet" (1.4-0.3) samples compared. It is found that the "intermediate" system produces the highest quality flakes with thickness of 2-10 nm, which the "wet" sample produces thicker flakes in the 10-200 $\mathrm{nm}$ range, interestingly a significant amount of oxidation is reported in the "dry" sample, 95 though this may not be surprising to a chemist as DMSO is often a principal component in organic oxidations e.g. the Swern reaction.

The $\mathrm{pH}$ of the solvent system is another parameter which can affect LPE. Saturated NMP solutions of $\mathrm{NaOH}$ have been used to exfoliate BP, the basic-NMP exfoliated BP has a large negative zeta potential of $-30.9 \mathrm{mV}$ compared to only $-19.7 \mathrm{mV}$ for standard NMP exfoliated BP implying a more stable colloid is created. The basic-NMP exfoliation makes it possible redisperse the nanosheets in water to form stable suspension. It also appears that basic-NMP yields more few-layer flakes, compared to standard NMP. ${ }^{96}$

Small molecules may also be added to the solvent to aid exfoliation, ${ }^{94,} 97$ phytic acid an organic molecule rich in hydroxyl and phosphate carboxyl functional groups was found to be a promising additive during exfoliation in DMF, increasing BP solubility and producing large nanosheets. ${ }^{97}$ Glucose was also found to be an effective additive, suggesting that hydroxyl groups may play an important role. ${ }^{97}$ Phytic acid has also been added to NMP to produce BP quantum dots $~ 3.4$ $\mathrm{nm}$ in diameter. ${ }^{94}$ It is not clear whether the dramatic difference in sheet size between these two studies is a result of the differences in solvent or if the smaller particles seen in NMP are a result of the longer sonication time and different centrifugation procedure used, such difficulties in comparison between similar studies are unfortunately common in the LPE literature.
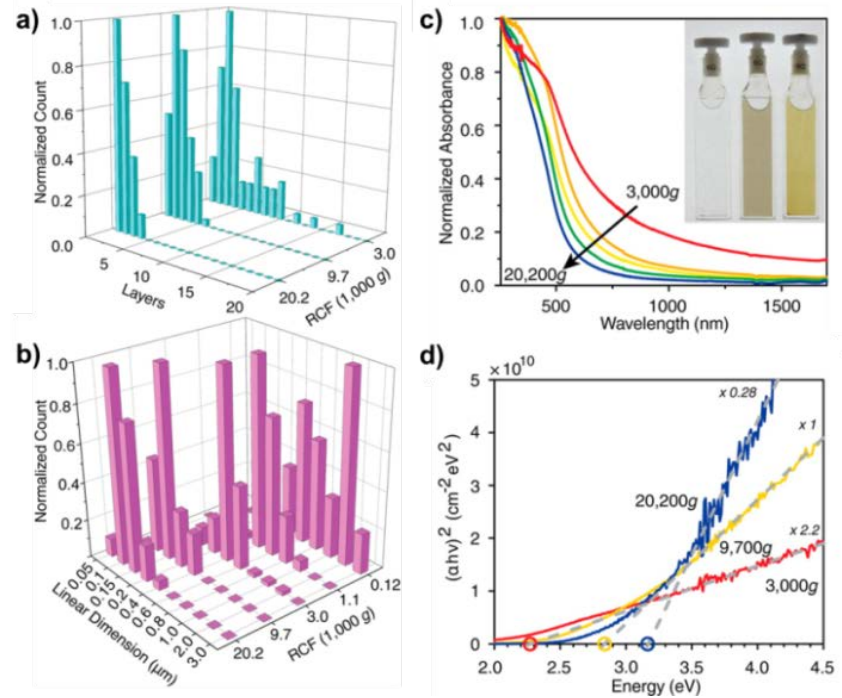

Figure 4. Size selection of LPE BP by centrifugation. (a-b) Size analysis of suspensions centrifuged at different relative centrifugal force (RCF) demonstrating that higher RCF values result in thinner flakes and smaller latera
dimensions. (c) Absorbance spectra and (d) direct Tauc plots of suspensions subjected to different RC.Fs. demnnstrating the size dependent optical properties of BP. Adanted with nermission from Woomer et al. ACS Nano 2015, 9 (9), 8869 8884 Copyright 2016 American Chemical Society.

It has been observed the LPE BP is considerably more stable than mechanically exfoliated material by techniques such as AFM (Figure 5) and XPS. ${ }^{49,86}$ It is likely that the solvents and or ligands involved in exfoliation play a role in protecting the exfoliated flakes, for example Coleman and co-workers have postulated that $\mathrm{CHP}$ forms a solvation shell which protects against oxygen and water. ${ }^{49}$ The fact the LPE flakes are coated in a layer of organic molecules is not entirely advantageous, for example in situations requiring high electron mobility LPE material is likely to perform worse than mechanically exfoliated material due to the need to pass current across flake boundaries, hampered by insulating organic ligands. ${ }^{86}$ However, there do appear to be a number of other applications that LPE flakes are well suited to including in sensors and in polymer composites. ${ }^{45,49,92,98-101}$ 
a. Mechanical exfoliation

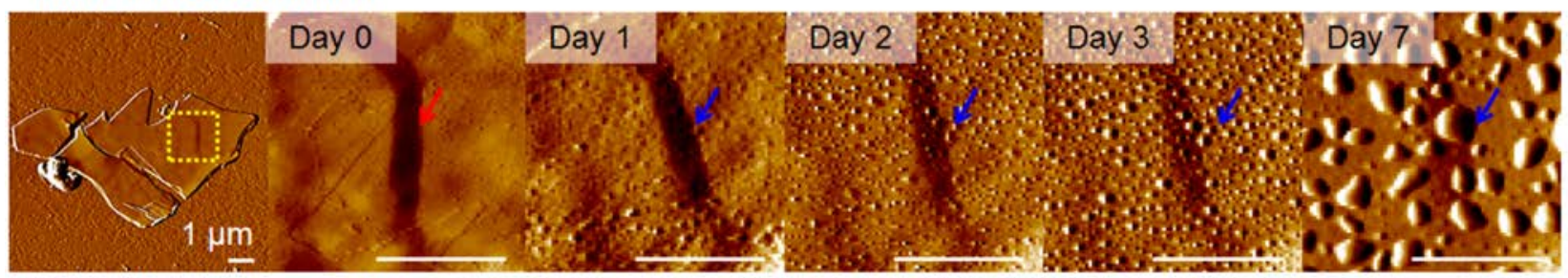

b. Liquid exfoliation in NMP

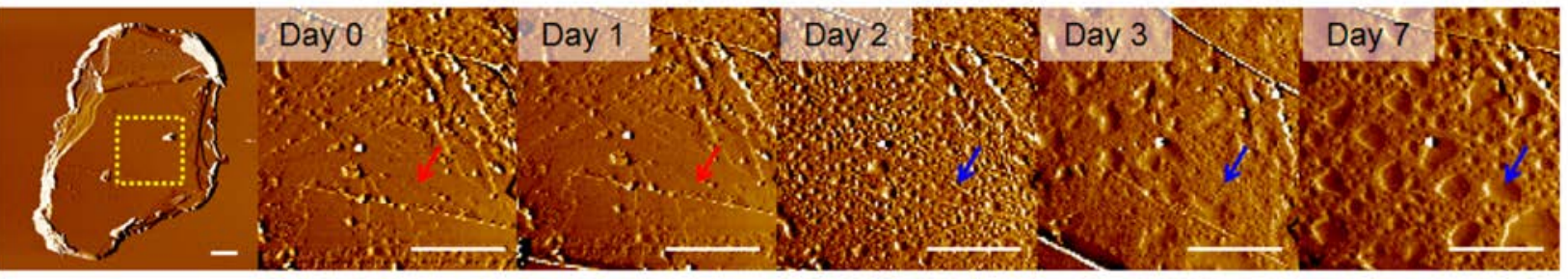

c. Mechanical exfoliation with dipping in NMP

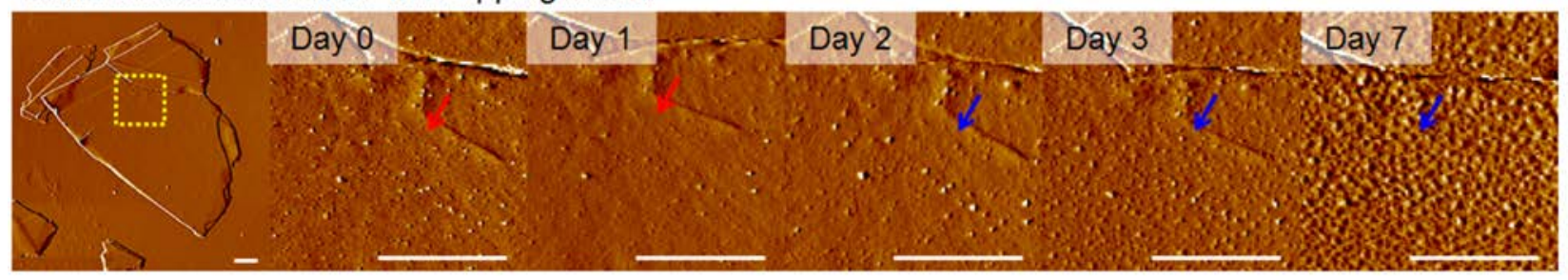

Figure 5: AFM Height profile time study from Kang et al. of BP flakes prepared by (a) mechanical exfoliation, (b) LPE in NMP, and (c) mechanical exfoliation followed by $1 \mathrm{~h}$ submersion in NMP. The leftmost image shows the entire flake, and the images progressing to the right show magnified views immediately after exfoliationup to 7 days in ambient conditions. Structural deformations (i.e., apparent bubbles) are observable on the mechanically exfoliated sample after 1 day and on the rest of the samples after 2 days. All scale bars are $1 \mu \mathrm{m}$. Reproduced with permission from Kang et al. ACS Nano 2015, 9 (4), 3596-3604. Copyright 2016 American Chemical Society.

The wide range of solvents investigated to-date for LPE of $B P$ is, in part, a reflection on the fact that there is, in our opinion, currently a lack of the understanding to rationally design LPE experiments. Identifying the key descriptors relevant to exfoliation will ultimately lead to more efficient progress in the field. It has been suggested that a close match between the cohesive energies of the solvent and the exfoliated materials, quantified by the Hansen solubility parameter approach, is a useful indicator of a good solvent system. ${ }^{102}$ However, this approach identifies a good solvent for colloidal stability and may not necessarily identify the best solvent for achieving few layer material or for preservation of the properties of the material. Computational studies may be able to help guide the discovery of optimised solvents for LPE. ${ }^{103}$ Exfoliation of BP was modelled using $a b$ initio molecular dynamics simulations. It was found that the shape of the solvent molecules is important, a 'solvent wedge' is involved in forcing layers apart and planar molecules increase the effectiveness of this process. ${ }^{104}$

The intercalation chemistry of layered compounds, where metal ions are inserted into the van der Waals space between layers, is rich. ${ }^{105-107}$ Indeed, this has been exploited in the use of $n$-butyl lithium as a chemical exfoliant via lithium insertion for producing 2D transition metal dichalcogenides such as $\mathrm{MoS}_{2}$ and $\mathrm{WS}_{2},{ }^{108-110}$ and similarly the use of iodine chloride and iodine bromide to produce graphene from graphite. ${ }^{111}$ As yet, and to the best of our knowledge, intercalative lithiation per se has not been applied to the synthesis of 2D BP and furthermore it seems unlikely that this route will be best suited to BP; although alkali metals can intercalate into the structure, they are so reactive with phosphorus that homogeneous intercalation without perturbation of the layer structure is thus refractory. ${ }^{112}$ Indeed, striking morphological changes associated with $\mathrm{Li}$ intercalation into BP have been observed in SEM of by Winter and co-workers potentially associated with the formation of $\mathrm{Li}_{3} \mathrm{P} .{ }^{113}$

\subsection{Liquid-phase exfoliation in aqueous media}

The quality of the exfoliated materials is not the only factor which will influence solvent choice. The ideal solvent would be cheap and non-toxic, and compatible with the envisaged applications of the nanosheets. For this reason LPE in aqueous solutions has recently become of considerable interest. ${ }^{75,114}$

Aqueous solutions have a number of advantages over organic solvents; not only is water cheaper and safer but unlike high boiling point organic solvents it readily evaporates post device fabrication leaving clean surfaces if no surfactants have been used to promote exfoliation. ${ }^{115}$ Aqueous solutions are especially attractive for applications where biocompatibility is necessary. ${ }^{116}$ While it is possible to exfoliate in an organic solvent and then re-disperse flakes in water, ${ }^{93,96,}$ 117 direct exfoliation in water is a simpler and more efficient approach. BP is highly hydrophilic and can thus be exfoliated in water with no surfactants. ${ }^{115}$ Milling red phosphorus with 
polyethylene glycol (PEG) has also been shown to produce PEGylated BP nanoparticles. ${ }^{118}$ To researchers familiar with mechanically exfoliated $\mathrm{BP}$, which is extremely sensitive to moisture, it may come as a surprise the LPE in water is feasible. The topic of BP degradation is addressed in section 3, however, at this point it is worth emphasising that aqueous BP flakes can be stable for weeks, ${ }^{116}$ making them suitable for a number of envisaged applications, such as photothermal treatment. In fact, in biomedical applications the eventual degradation of nanosheets into biocompatible ions is an advantage compared to other nanomedicine technologies where clearing is a concern. ${ }^{116,119,120}$

Surfactants are frequently employed to assist LPE in water. ${ }^{121,122}$ Surfactant assisted LPE of BP in water was first reported by Hersam et al. using a 2 wt.\% SDS solution. ${ }^{123}$ It is possible to achieve superior concentrations in aqueous SDS solutions than in NMP. The surfactant is found to improve stability compared to water alone and it is also reported that the flakes produced are thinner than those produced by exfoliation in anhydrous organic solvents. ${ }^{123}$ We used the surfactant Triton X-100 for BP exfoliation, producing sheets less than $20 \mathrm{~nm}$ thick and $100-200 \mathrm{~nm}$ in lateral dimensions, ${ }^{45}$,

50 the surfactant is shown to play a key role in slowing the degradation process (see section 3), though it is clear the materials do have a 'shelf life' in water. ${ }^{50}$ Titanium sulfonate ligands have also been demonstrated to bind to BP flakes and reduce degradation in water significantly. ${ }^{117}$ Currently one of the major areas of interest for aqueous BP is for photothermal ablation of cancer cell, ${ }^{118} 117$ BP suspensions can absorb radiation of near-infrared wavelengths and can significantly increase local temperatures proximal to the flakes. For example PEGylated BP nanoparticles are able to increase the temperature of a solution by $36^{\circ} \mathrm{C}$, remain functional for at least 8 days and show long retention times in tumours but are easily removed from the liver and kidney. ${ }^{118}$

\subsection{Shear mixing}

One of the major attractions of LPE is that is that it is able to produce a huge number of flakes compared to mechanical exfoliation. However, ultrasonication of layered materials is typically reported on the 10 or $100 \mathrm{mg}$ scale. ${ }^{82,87}$ To move towards commercialization it is necessary to move to the gram or even kilogram scale pilot and production batch scales. Replacing bath or probe ultrasonication with shear mixing for the exfoliation of $2 \mathrm{D}$ materials has been identified as a promising route as the use of a rotating blade to generate either turbulent or laminar shear in solutions can be scaled to industrially relevant volumes. Coleman et. al. have successfully demonstrated the production of graphene suspensions on the litre scale using shear mixing methods. ${ }^{124,} 125$ Nanosheets can be produced using both dedicated rotor/stator high-shear mixers (laminar regime) and kitchen blenders (turbulent regime) (Fig. 1c). ${ }^{124,125}$ Shear exfoliation has been shown to be a considerably more energy-efficient method of exfoliating graphite than sonication. ${ }^{124}$

Shear mixing has been used to exfoliate black phosphorus in NMP (Fig. 1d). ${ }^{87}$ It is found that this approach is sensitive to the quality of the bulk BP starting material. High purity BP with large single crystals could not be exfoliated by shear mixing alone, however, lower quality polycrystalline samples containing trace amounts of red phosphorus could be exfoliated effectively, suggesting that grain boundaries or defects are required. ${ }^{87}$ Initial bath sonication followed by shear mixing was found to be an effective method of exfoliating the higher quality single crystal material. ${ }^{87} \mathrm{Li}$ et. al. also report successful shear exfoliation of BP in NMP in both laminar and turbulent regimes but do not report needing defect rich BP. ${ }^{126}$ $\mathrm{BP}$ quantum dots with an average lateral diameter of $2.3 \mathrm{~nm}$ have been prepared using turbulent shear mixing. Here, a range of solvents were investigated showing a trend for decreasing particle size with increasing surface tension. ${ }^{92}$

\subsection{Processing liquid phase exfoliated flakes}

A significant advantage of LPE is the ease with which the resulting suspensions are compatible with various solution phase processes. Centrifugation is perhaps the most widely employed post-exfoliation processing; initial suspensions of LPE nanosheets are typically highly polydisperse. Given the size-dependent properties of nanomaterials it is typically desirable to isolate a narrow size distribution for a given application. Centrifugation is commonly employed to perform size selection of LPE nanosheets, ${ }^{127,}{ }^{128}$ with the recent development of multi-step cascade centrifugation protocols allowing $\mathrm{WS}_{2}$ dispersions containing up to $75 \%$ monolayer flakes to be produced. ${ }^{91}$

Centrifugation has been widely used to purify LPE BP nanosheets (Fig $1 \mathrm{~b}$ and Fig 3), ${ }^{87,88,96,99}$ for example samples which were either $\sim 7$ layers thick and $50 \mathrm{~nm}-1 \mu \mathrm{m}$ in diameter or $\sim 3$ layers thick and $50-300 \mathrm{~nm}$ in diameter were isolated from the same formamide suspension of LPE BP by employing different centrifugation speeds. ${ }^{88}$ Selecting flakes by centrifugation can directly affect performance in application, for example humidity sensors made of LPE flakes showed the best performance when the highest centrifuge speeds were employed to give the thinnest flakes and smallest lateral dimensions. ${ }^{99}$

A number of processing routes can be used to incorporate LPE flakes into composite materials. Vacuum filtration of nanosheet suspensions is widely used to produce membranes of re-stacked flakes. ${ }^{129-131}$ Vacuum filtration of a mixtures of LPE BP and graphene oxide was used to produce a composite film which was then annealed to reduce the graphene oxide, the resulting material is used as a lithium ion battery anode, showing high gravimetric capacity, good rate capability, and 
a)

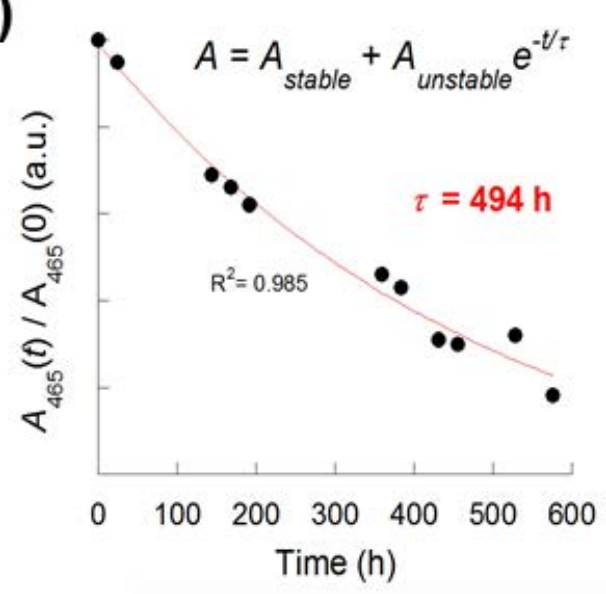

b)

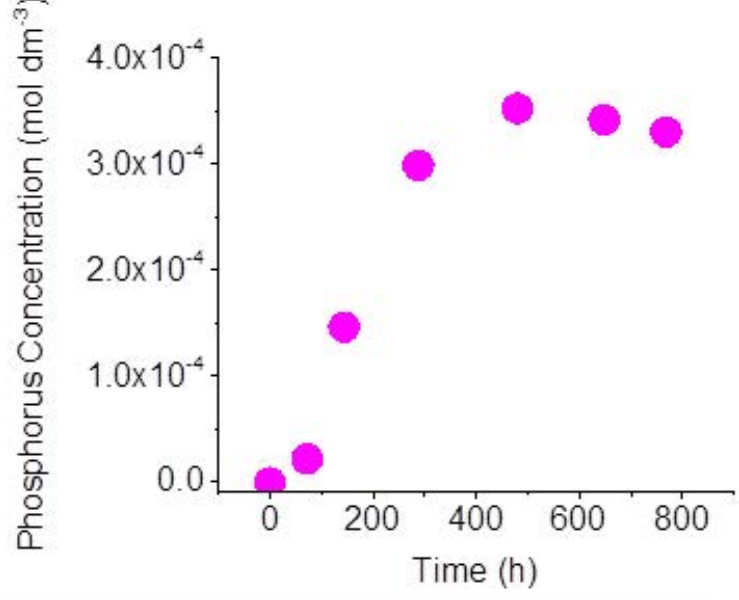

c)

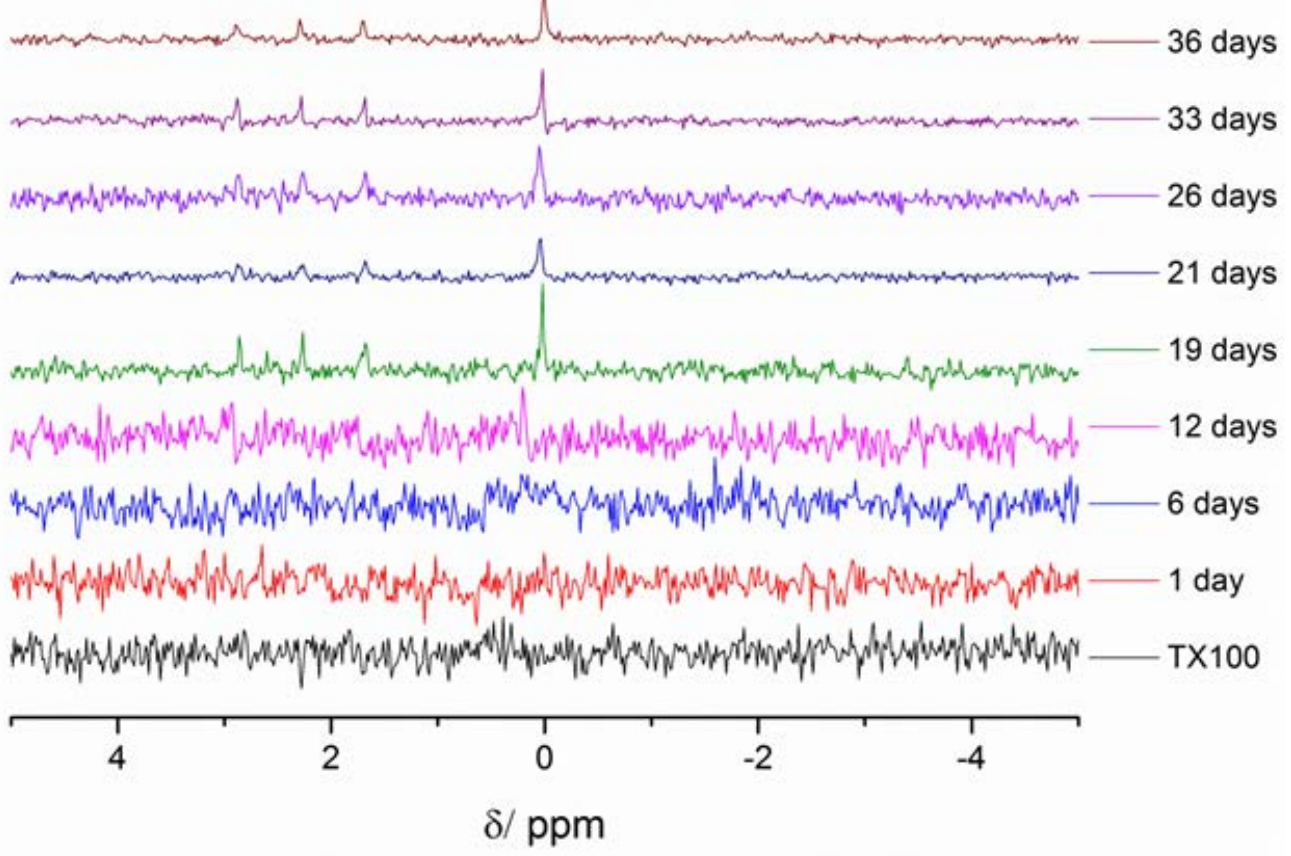

Figure 6: Spectroscopic assessment of the stability of exfoliated black phosphorus nanosheets in $1 \% w / v$ aqueous Triton X-100 solution from Brent et al. (a) UV-Vis absorbance spectroscopy time study of the stability of BP nanosheets in $1 \% \mathrm{w} / \mathrm{v}$ aqueous Triton $\mathrm{X}-100$. The change in absorbance at $465 \mathrm{~nm}$ is used as an indicator of the amount of black phosphorus remaining in solution and can be fitted to the empirical function $\frac{A_{t}}{A_{0}}=A_{\text {stable }}+A_{\text {unstable }} e^{-t / \tau}$. The mean observed lifetime of the nanosheets in solution is estimated to be almost $500 \mathrm{~h}$ using this method. (b) ICP-OES time study of the absolute concentration of phosphorus released into solution from degradation of the BP nanosheets in $1 \% w / v$ aqueous Triton X-100, showing a plateau-like profile. $\mathbf{c}$, ${ }^{31} \mathrm{P}$ NMR time study of the phosphorus species released into solution from degradation of sheets in $1 \% \mathrm{w} / \mathrm{v}$ aqueous Triton X-100 over a period of 36 days ( $864 \mathrm{~h}$ ). The black trace is the NMR spectrum of $1 \% \mathrm{w} / \mathrm{v}$ aqueous Triton X-100, which contains no phosphorus species. Adapted from Brent et al. RSC Adv. 2016, 6, $86955-$ Published by The Royal Society of Chemistry.

good retention of performance during cycling. ${ }^{132} \mathrm{~A}$ LangmuirBlodgett trough has been used to coat NMP exfoliated BP flakes onto a substrate, achieving compact arrangement of nanosheets over a larger area. ${ }^{133}$ LPE flakes and polymers can be combined in a solvent in which both are soluble. ${ }^{41,134,135} \mathrm{BP}$ flakes dispersed in chloroform can be mixed with polycarbonate, the resulting polymer composite material was used for pulse generation in an all-fibre laser, the polymer matrix also appears to reduce $\mathrm{BP}$ degradation. ${ }^{98} \mathrm{BP}-$ polyvinylchloride composite films have also been investigated,
0.3 vol\% loading of BP is shown to double the strength and increase tensile toughness six-fold. ${ }^{49}$ It is also possible to inkjet print nanosheet suspensions, ${ }^{79,136}$ and it seems likely that this technique will be applied to the processing of LPE BP in the future.

\subsection{Other top-down approaches}

Mechanical exfoliation and liquid phase exfoliation are both examples of top-down approaches, in which layered bulk material is broken down into thin nanosheets. Another 
interesting top down approach is the use of thermal sublimation to thin few layer BP. At $500 \mathrm{~K}-600 \mathrm{~K} \mathrm{BP}$ is observed to sublime one atomic layer at a time, and this approach can be used to produce large thin flakes with monolayers larger than $200 \mathrm{um}^{2}$ prepared by heating BP under an $\mathrm{N}_{2}$ atmosphere. ${ }^{137}$ However, this pathway suffers from many of the draw backs associated with mechanical exfoliation, such as the need to identify and transfer individual flakes and incompatibility with solution processing. BP has also been exfoliated electrochemically, producing flakes 5-10 $\mu \mathrm{m}$ in diameter and 1-5 $\mathrm{nm}$ thick. ${ }^{138}$

\subsection{Bottom up approaches}

Bottom-up syntheses represent an alternative approach to producing 2D materials where, instead of delaminating existing layered crystals, nanocrystals are formed from atomic or molecular building blocks. Whilst there have been some successful demonstrations of solution phase chemical reactions producing 2D nanosheets, ${ }^{139-141}$ chemical vapour deposition (CVD) has emerged as the most promising bottomup route to $2 \mathrm{D}$ materials. However, to the best of our knowledge there are no successful reports of CVD of 2D BP at this juncture.

One interesting result is the report that red phosphorus can converted to BP nanosheets through a solvothermal reaction in ethanol involving a solid-vapour-solid transformation. The flakes produced are polycrystalline and defective. However, it may be possible to grow higher quality BP for an ethanol/phosphorus vapour in the future. ${ }^{88}$ Another successful demonstration of bottom-up BP synthesis used pulsed laser deposition (PLD), with films from 2-10 nm thick produced, however, the BP deposited by this route is a highly disordered amorphous form. Nonetheless the material shows a thickness-dependent band gap and the expected Raman signature of BP. ${ }^{142}$

\subsection{Summary}

Liquid phase exfoliation is currently the most promising approach for simple and scalable production of BP nanosheets. Mechanical exfoliation, while useful for fundamental studies, is completely unscalable and to-date there are no reports regarding CVD growth of BP. If commercialisation of BP based technologies is the ultimate goal then shear mixing has advantages over ultrasonication in terms of scalability although the latter approach has been more widely studied in the research community to-date. The field of liquid phase exfoliation still in its infancy and consequently lacks strict standards for reporting experimental procedures and characterising products, though these are emerging. This means that direct comparison between different experimenta studies can often be challenging. However, knowledge of the conditions that produces 2D BP of a certain thickness is extremely useful for device manufacture; Dresselhaus and coworkers state, for instance, that 2D BP with a thickness in the range of $4-10 \mathrm{~nm}$ would strike the best balance between carrier mobility and on-off current ratio for devices operating in the multigigahertz frequency range. ${ }^{143}$ We summarise the LPE routes discussed in this article and the typical thicknesses of nanosheets produced using these approaches in Table 1. It should be noted that by the development of careful centrifugation-purification protocols (e.g. as in Woomer et al. ${ }^{87}$ ) that further size selection could be achieved for many of these 'as-published' processes. An example recently is the development of cascade centrifugation by Coleman. ${ }^{91}$

\section{Challenges in processing of few-layer black phosphorus}

\subsection{Environmental Instability}

Despite being the most thermodynamically stable allotrope of phosphorus in the bulk form, degradation of ultrathin samples in the presence of water and oxygen has been widely observed since its isolation. Long-term stabilisation of phosphorene has been demonstrated via encapsulation of solid films; ${ }^{39,40,144,145}$ however minimisation of oxidation, especially in solution remains an ongoing challenge. ${ }^{49,50,82}$ Flake degradation is associated with reduced transistor performance due to strong p-type doping of the device and a reduction in ON/OFF ratio of several orders of magnitude. ${ }^{146}$ This instability and the lack of vapour deposition routes to large-area nanosheet films are likely to prove the largest obstacles to real-world exploitation of 2D BP's potential.

First principles calculations indicate that pristine phosphorene does not interact strongly with $\mathrm{H}_{2} \mathrm{O}$ and that oxidation proceeds via initial dissociation of $\mathrm{O}_{2}$ on the nanosheet surface, followed by increasing hydrophilicity and subsequent reaction with $\mathrm{H}_{2} \mathrm{O}$. This has recently been confirmed experimentally, with recent results showing that in the first instance, flake etching is driven by the availability of $\mathrm{O}_{2}$, even in aqueous environments. ${ }^{147}$ Degradation of mechanically exfoliated flakes is also a photo-assisted process $^{148}$ and begins immediately upon exposure to ambient conditions, resulting in significant disruption of the sheet structure $^{145,148}$ and eventual etching of the flake. ${ }^{149}$ The breakdown products include phosphates/phosphoric acids ${ }^{49,50 \text {, }}$ 145,148 and accompanying defects and oxygen adatoms. The acidic products coalesce into droplets which, after 1 hour may be observed under atomic force or optical microscopy ${ }^{149}$ and after several days can be seen with the naked eye.

Encapsulation of micromechanically exfoliated flakes has been shown to greatly increase the longevity of phosphorene flakes and preserve the performance of devices. ${ }^{40}, 144,145$ Encapsulation also minimises the electronic disruption caused by phosphorene's strong interactions with atmospheric molecules. ${ }^{44,150}$ This has typically been carried out using conventional inorganic or polymeric insulators to provide a physical barrier to the ingress of gaseous contaminants. As well as providing increased stability, these materials have the advantage of also providing an in-built dielectric layer, thereby further improving transistor performance.

Hersam and co-workers initially investigated the use of $\mathrm{AlO}_{\mathrm{x}}$ capping layers fabricated by ALD and found that 
encapsulation both improves device performance and that the properties were preserved over the 7 day study. ${ }^{145}$ Further work from Akinwande et al found that device performance begins to diminish over time, ${ }^{39}$ indicating that alumina is not able to fully protect the sheet surface. This has been improved via the inclusion of a layer of hydrophobic fluoropolymer to add an additional barrier. ${ }^{39}$ Hexagonal boron nitride ( $\left.h-\mathrm{BN}\right)$ has also been shown to allow long-term protection of the phosphorene layer without negatively impacting the electronic properties of the device. ${ }^{40}$ As $h$-BN is itself a 2D material, encapsulation has the additional benefits of allowing the formation of van der Waals (vdW) heterostructures and the creation of BP/h-BN/BP sandwiches which keep phosphorene layers electronically decoupled from one another, thereby preserving few-layer properties even in restacked films. ${ }^{58,151}$ If gas-phase routes to phosphorene can be developed it will be essential to devise methods for encapsulation in situ in order to minimise atmospheric exposure; as boron nitride is readily deposited from the gas phase ${ }^{152,153}$ it is surely a strong candidate for incorporation into phosphorene syntheses.

Due to the continuing lack of bottom-up routes, various groups have focussed on the scalable exfoliation of phosphorene and its stability in solution. ${ }^{45,49,50,123}$ Coleman et al were among the first to study the breakdown of phosphorene nanosheets and found the organic solvent used to disperse the material imparts some stability by providing an imperfect barrier to moisture ingress. ${ }^{49}$ It was determined that oxidation proceeds from sheet edges and defect sites and is associated with a concomitant decrease in $\mathrm{pH}$ due to production of phosphorus and/or phosphoric acid. This study postulated that the most likely reaction product was $\mathrm{H}_{3} \mathrm{PO}_{3}$. it has also been suggested that insulating $\mathrm{P}_{2} \mathrm{O}_{5}$ is the most stable product and that the formation of a protective oxide may prevent further oxidation of underlying layers, ${ }^{154}$ however given the numerous reports of film etching discussed above, this seems unlikely to be the case in ultrathin black phosphorus.

Under optimised conditions, the organic dispersions are stable for up to 100 times longer than mechanically exfoliated flakes, a timeframe comparable with initial attempts to encapsulate solid phosphorene nanosheets. ${ }^{39,49} \mathrm{NMP}$ and CHP are effective dispersants, in part due to their strong interactions with the nanosheet surface. This has been exploited to allow redispersion of NMP-exfoliated phosphorene in aqueous solution, with bound NMP providing some protection against oxidation in the short term. ${ }^{96}$ Coleman and colleagues also explored the direct, surfactantassisted exfoliation of phosphorene in water, finding that an aqueous sodium cholate solution afforded less stability than organic solvents, though the dispersions were again considerably more chemically stable than exposed micromechanically exfoliated flakes. ${ }^{49}$ Hersam and coworkers then showed that aqueous surfactant-based phosphorene sols can also be highly colloidally stable. ${ }^{123}$ More recent studies by the authors indicate that through judicious choice of surfactant and careful degassing of solvent, aqueous-based sols can exhibit chemical stability which matches or exceeds that of organic solvents. ${ }^{50}$ Orthophosphate $\left(\mathrm{PO}_{4}{ }^{-}\right)$was found to be the only phosphorus species released by phosphorene breakdown, in agreement with previous predictions. ${ }^{45,49}$ In organic media, it appears that phosphorene oxidation proceeds until dissolved water in the sol is depleted. In aqueous solution, there is essentially an infinite reservoir of $\mathrm{H}_{2} \mathrm{O}$ yet degradation appears to be limited to around $80 \%$ of total mass of phosphorene. It is likely that degradation proceeds to such a point that the remaining flake is encapsulated within the surfactant micelle and is more fully protected (Figure 6). ${ }^{45}$ Further work, correlating nanosheet size and mass of dispersed material with the critical micelle concentration of several surfactants would shed more light on this problem, and potentially allow for more rational design of stabilising molecules.

\subsection{Other Challenges}

Liquid exfoliation is a potentially scalable process for 2D BP production however nanosheet grain size is inherently limited by the size of the starting material and is consistently reduced when going to longer exfoliation regimes as are needed to optimise the uniformity of the dispersed material. Further to this, phosphorene's electronic properties are highly orientation dependant; ${ }^{14}$ therefore optimised devices will require highly-orientated nanosheet films. Non-random nanosheet deposition from solution remains problematic, significantly reducing phosphorene's utility. It is therefore necessary to develop methods by which highly ordered films may be prepared from solution or, more preferably via gaseous deposition. Vapour-transport methods are held back by the high pressures required to force preferential formation of black phosphorus over other phosphorus allotropes, ${ }^{18}$ although the production of CVD diamond shows that CVD does not necessarily favour the thermodynamically stable allotrope of a material under all deposition condition. Progress has been made via a two-step process to deposit an ultrathin layer of red phosphorus, followed by high pressure treatment to form a black phosphorus film. ${ }^{155}$ Although this is a significant improvement over previous methods, the films are non-continuous and relatively thick, often several 100 $\mathrm{nm}$. It is possible that, as with graphene and $\mathrm{MoS}_{2}$, nanosheet CVD growth on suitable liquid metals ${ }^{156,157}$ or on highly lattice-matched substrates ${ }^{158,159}$ may promote isolation of black phosphorus sheets. For example, recently isolated $\mathrm{SnS}$ nanosheets ${ }^{74}$ have similar lattice parameters to black phosphorus ${ }^{160}$ and past syntheses of bulk black phosphorus have utilised molten bismuth ${ }^{161}$ and mercury fluxes; ${ }^{162}$ it may be possible to utilise these materials in the synthesis of its $2 \mathrm{D}$ analogue, phosphorene. 
Table 1: Summary of solvents used for LPE and the resulting thickness of 2D BP.

\begin{tabular}{c|c|c}
\hline Solvent & Sheet Thickness & Reference \\
\hline Organic & $1-5 \mathrm{~nm}$ & \\
NMP & $<10 \mathrm{~nm}$ & 81 \\
NMP & $17.6 \mathrm{~nm}$ & 82 \\
NMP & $2.8-5.3 \mathrm{~nm}$ & 123 \\
NMP/sat. NaOH & $9.4 \pm 1.3 \mathrm{~nm}$ & 96 \\
CHP & $15-20 \mathrm{~nm}$ & 89 \\
DMSO & $<10 \mathrm{~nm}$ & 86 \\
DMF & & \\
Aqueous & $2 \mathrm{~nm}$ & 116 \\
Distilled water & $<20 \mathrm{~nm}$ & 45,50 \\
1\% aq. TX-100 & $4.5 \mathrm{~nm}$ & 123 \\
2\% aq. SDS & & \\
Ionic Liquids & & 89 \\
[BMIM][TfO] & $8.5-12.8 \mathrm{~nm}$ & 89 \\
[HOEMIM][TfO] & $3.6-8.9 \mathrm{~nm}$ & \\
\hline
\end{tabular}

\subsection{Applications}

Phosphorene's anisotropic behaviour lends itself to use as a thermoelectric material. ${ }^{21}$ Strong thermoelectric performance requires both high electrical and low thermal conductance such a combination is rare due to a strong correlation between the two. ${ }^{163}$ Phosphorene's potential in this field is greatly enhanced by the orthogonal preferred directions of electrical and thermal conductivity; ${ }^{47}$ with thermal conduction more efficient long the zigzag direction and electrical conduction preferring the armchair direction. ${ }^{47}$ Performance may be further improved by applying strain ${ }^{46,164}$ or doping, however even pristine monolayers are calculated to provide a substantial energy conversion efficiency (up to 20\%) even at room temperature, which compares favourably with currently available technology.

Density functional theory (DFT) calculations have shown that phosphorene monolayers also exhibit rapid, anisotropic $\mathrm{Na}$ diffusion, and high conductivity and stability, therefore showing great promise as a potential anode material for alternative alkali metal ion batteries. ${ }^{42}$ Phosphorene's theoretical capacity for sodium ions is predicted to be higher than that of $\mathrm{MoS}_{2}$ and commercial graphite ${ }^{42}$ and phosphorene-graphene hybrids employed as sodium ion anodes have displayed a specific capacity of $2440 \mathrm{~mA} \mathrm{~h} \mathrm{~g}{ }^{-1}$ (calculated from mass of phosphorus only). ${ }^{17}$ Graphene is usually added to provide greater mechanical robustness and therefore increase the overall cycling stability. ${ }^{17}$ The formation of phosphorene/graphene heterostructures can also produce a synergistic effect which increases the Li binding energy with the result of significantly improving the safety and reversibility of Li-ion batteries. ${ }^{43}$

2D BP has potential for producing effective vdW heterostructures with other 2D materials in stack-type configurations where the flakes are overlayed or assembled between bulk interfaces to produce engineered materials with exotic properties. A $\mathrm{p}-\mathrm{n}$ junction that is able to operate in photovoltaic mode based on a 2D BP / $\mathrm{MoS}_{2}$ heterostructure has been reported by Ye and co-workers, with an external quantum efficiency (EQE) of $0.3 \% .{ }^{165}$ The power conversion efficiency of photovoltaic devices based on $2 \mathrm{D} \mathrm{BP} / \mathrm{MoS}_{2} \mathrm{vdW}$ heterostructures has been predicted to be as great as $18 \%$ based on DFT calculations. ${ }^{166}$ Tunnelling field effect transistors (TFETs) have been Hine and Constaninescu based on a 2D $\mathrm{BP} / \mathrm{hBN} / 2 \mathrm{D}$ BP stack. ${ }^{151}$ Kuiri et al have made quantum capacitance and conductance measurements on hBN /2D BP heterostructures in a dual-gated FET. ${ }^{167}$ Very recently Huber and co-workers have reported $\mathrm{SiO}_{2} / \mathrm{BP} / \mathrm{SiO}_{2}$ type interfaces where the phonon coupling between $\mathrm{BP}$ and $\mathrm{SiO}_{2}$ can be used for ultrafast polariton switching. ${ }^{168}$ The incorporation of 2D BP into vdW heterostructures may potentially be the best way to exploit the properties of the material as some degree of protection from atmospheric oxidation can be imparted by vertical entombment. ${ }^{58}$

\section{Conclusions and Outlook}

Phosphorene, the monolayer 2D synthetic allotrope of black phosphorus, has remarkable properties, including a tuneable band gap, high carrier mobility and anisotopic optoelectronic properties due to its armchair/zig-zag structure. It is also, despite its inherent instability toward water and other oxidising agents, one of the most stable 2D elemental analogues beyond graphene and can be processed using a range of techniques, from scotch tape to LPE. There are synthetic challenges when handling $2 \mathrm{D} B \mathrm{~B}$, arising principally from its susceptibility to oxidation but research efforts have been somewhat successful in circumventing these, and indeed water-stable dispersions are now possible due to an understanding of the chemistry and breakdown mechanisms of the material.

It is likely that a number of research directions will continue in the short to medium term that will include finding better ways to stabilise the material in solution based on the understanding of its chemistry. Fluorinated surfactants, for instance, have been shown to effectively protect nanostructures from oxygen ingress to their surface and this could be an effective way to passivate BP in order to extend its lifetime in solution. ${ }^{169}$ The other side of this coin is that the instability of BP may be exploited to produce biocompatible systems that break down to release phosphate-type species in a controlled manner in situ for example, in bone healing applications. Indeed, only with a good understanding of the chemistry of this interesting material can it be exploited to its full potential in a range of applications as a semiconductor in a future electronics industry, which we envisage as an exciting long-term prospect. 


\section{Acknowledgements}

BD acknowledges the support of EPSRC grant number $\mathrm{EP} / \mathrm{N} 010345 / 1$ (Engineering van der Waals heterostructures: from atomic level layer-by-layer assembly to printable innovative devices). E.A.L thanks the EPSRC for the award of a Doctoral Prize. All data underlying this study are cited in the references.

\section{Notes and references}

1. G. R. Bhimanapati, Z. Lin, V. Meunier, Y. Jung, J. Cha, S. Das, D. Xiao, Y. Son, M. S. Strano, V. R. Cooper, L. Liang, S. G. Louie, E. Ringe, W. Zhou, S. S. Kim, R. R. Naik, B. G. Sumpter, H. Terrones, F. Xia, Y. Wang, J. Zhu, D. Akinwande, N. Alem, J. A. Schuller, R. E. Schaak, M. Terrones and J. A. Robinson, ACS Nano, 2015, 9, 11509-11539.

2. A. Gupta, T. Sakthivel and S. Seal, Progress in Materials Science, 2015, 73, 44-126.

3. S. Z. Butler, S. M. Hollen, L. Cao, Y. Cui, J. A. Gupta, H. R. Gutiérrez, T. F. Heinz, S. S. Hong, J. Huang, A. F. Ismach, E. Johnston-Halperin, M. Kuno, V. V. Plashnitsa, R. D. Robinson, R. S. Ruoff, S. Salahuddin, J. Shan, L. Shi, M. G. Spencer, M. Terrones, W. Windl and J. E. Goldberger, ACS Nano, 2013, 7, 2898-2926.

4. H. Chandrasekar, K. B. B, K. K. Vaidyuala, S. Suran, N. Bhat, V. Manoj and S. Raghavan, Nanotechnology, 2015, 26, 425202

5. B. Anasori, C. Shi, E. J. Moon, Y. Xie, C. A. Voigt, P. R. C. Kent, S. J. May, S. J. L. Billinge, M. W. Barsoum and Y. Gogotsi, Nanoscale Horiz., 2016, 1, 227-234.

6. C. Xu, L. Wang, Z. Liu, L. Chen, J. Guo, N. Kang, X.L. Ma, H.-M. Cheng and W. Ren, Nat Mater, 2015, 14, 1135-1141.

7. L. Dou, A. B. Wong, Y. Yu, M. Lai, N. Kornienko, S. W. Eaton, A. Fu, C. G. Bischak, J. Ma, T. Ding, N. S. Ginsberg, L.-W. Wang, A. P. Alivisatos and P. Yang, Science, 2015, 349, 1518-1521.

8. Y.-H. Kim, H.-J. Kim, M. Osada, B.-W. Li, Y. Ebina and T. Sasaki, ACS Appl. Mater. Interfaces, 2014, 6, 19510-19514.

9. R. Fei and L. Yang, Nano Lett., 2014, 14, 28842889.

10. A. Acun, L. Zhang, P. Bampoulis, M. Farmanbar, A. v. Houselt, A. N. Rudenko, M. Lingenfelder, G. Brocks, B. Poelsema, M. I. Katsnelson and H. J. W. Zandvliet, J. Phys.: Condens. Matter, 2015, 27, 443002.

11. F.-f. Zhu, W.-j. Chen, Y. Xu, C.-I. Gao, D.-d. Guan, C.-h. Liu, D. Qian, S.-C. Zhang and J.-f. Jia, Nat Mater, 2015, 14, 1020-1025.

12. S. Balendhran, S. Walia, H. Nili, S. Sriram and M. Bhaskaran, Small, 2015, 11, 640-652.

13. R. F. Service, Science, 2015, 348, 490-492.

14. H. Liu, A. T. Neal, Z. Zhu, Z. Luo, X. Xu, D. Tománek and P. D. Ye, ACS Nano, 2014, 8, 4033-4041.
15. L. Li, Y. Yu, G. J. Ye, Q. Ge, X. Ou, H. Wu, D. Feng, X. H. Chen and Y. Zhang, Nat. Nanotechnol., 2014, 9, 372-377.

16. S. Cui, H. Pu, S. A. Wells, Z. Wen, S. Mao, J. Chang, M. C. Hersam and J. Chen, Nat Commun, 2015, 6, 8632.

17. J. Sun, H.-W. Lee, M. Pasta, H. Yuan, G. Zheng, Y. Sun, Y. Li and Y. Cui, Nat. Nanotechnol., 2015, 10, 980985.

18. P. W. Bridgman, J. Am. Chem. Soc., 1914, 36, 1344-1363.

19. P. W. Bridgman, J. Am. Chem. Soc., 1916, 38, 609612.

20. L. Shulenburger, A. D. Baczewski, Z. Zhu, J. Guan and D. Tománek, Nano Lett., 2015, 15, 8170-8175.

21. H. Zhou, Y. Cai, G. Zhang and Y.-W. Zhang, J. Mater. Res., 2016, 31, 3179-3186.

22. Q. Wu, L. Shen, M. Yang, Y. Cai, Z. Huang and Y. P. Feng, Phys. Rev. B, 2015, 92, 035436.

23. G. Wang, G. C. Loh, R. Pandey and S. P. Karna, Nanotechnology, 2016, 27, 055701.

$2424 . \quad$ R. B. Jacobs, J. Chem. Phys., 1937, 5, 945-953.

25

25. Y. Maruyama, S. Suzuki, K. Kobayashi and S. Tanuma, Physica B+C, 1981, 105, 99-102.

26. A. Brown and S. Rundqvist, Acta Cryst., 1965, 19, 684-685.

27. S. Lange, P. Schmidt and T. Nilges, Inorg. Chem., 2007, 46, 4028-4035.

28

28. T. Nilges, M. Kersting and T. Pfeifer, Journal of Solid State Chemistry, 2008, 181, 1707-1711.

29. S. H. Aldave, M. N. Yogeesh, W. Zhu, J. Kim, S. S. Sonde, A. P. Nayak and A. Deji, 2D Mater., 2016, 3, 014007.

3030 . J. Kim, S. Kwon, D.-H. Cho, B. Kang, H. Kwon, Y. Kim, S. O. Park, G. Y. Jung, E. Shin, W.-G. Kim, H. Lee, G. H. Ryu, M. Choi, T. H. Kim, J. Oh, S. Park, S. K. Kwak, S. W. Yoon, D. Byun, Z. Lee and C. Lee, Nat Commun, 2015, 6, 8294.

31 31. G. Schusteritsch, M. Uhrin and C. J. Pickard, Nano Lett., 2016, 16, 2975-2980.

32 32. A. N. Rudenko and M. I. Katsnelson, Phys. Rev. B, 2014, 89, 201408.

33. Z. Guo, H. Zhang, S. Lu, Z. Wang, S. Tang, J. Shao, Z. Sun, H. Xie, H. Wang, X.-F. Yu and P. K. Chu, Adv. Funct. Mater., 25, 7100.

34. K. F. Mak, C. Lee, J. Hone, J. Shan and T. F. Heinz, Phys. Rev. Lett., 2010, 105, 136805.

35. S. Kim, A. Konar, W.-S. Hwang, J. H. Lee, J. Lee, J. Yang, C. Jung, H. Kim, J.-B. Yoo, J.-Y. Choi, Y. W. Jin, S. Y. Lee, D. Jena, W. Choi and K. Kim, Nat Commun, 2012, 3, 1011.

36. K. Kang, S. Xie, L. Huang, Y. Han, P. Y. Huang, K. F. Mak, C.-J. Kim, D. Muller and J. Park, Nature, 2015, 520, 656-660. 37. W. Wu, D. De, S.-C. Chang, Y. Wang, H. Peng, J. Bao and S.-S. Pei, Appl. Phys. Lett., 2013, 102, 142106. 38. L. Kou, C. Chen and S. C. Smith, J. Phys. Chem. Lett., 2015, 6, 2794-2805. 
39. J.-S. Kim, Y. Liu, W. Zhu, S. Kim, D. Wu, L. Tao, A. Dodabalapur, K. Lai and D. Akinwande, Sci. Rep., 2015, 5, 8989.

40. A. Avsar, I. J. Vera-Marun, J. Y. Tan, K. Watanabe, T. Taniguchi, A. H. Castro Neto and B. Özyilmaz, ACS Nano, 2015, 9, 4138-4145.

41. X. Zhang, H. Xie, Z. Liu, C. Tan, Z. Luo, H. Li, J. Lin, L. Sun, W. Chen, Z. Xu, L. Xie, W. Huang and H. Zhang, Angew. Chem. Int. Ed., 2015, 54, 3653-3657.

42. V. V. Kulish, O. I. Malyi, C. Persson and P. Wu, Phys. Chem. Chem. Phys., 2015, 17, 13921-13928.

43. G.-C. Guo, D. Wang, X.-L. Wei, Q. Zhang, H. Liu, W.-M. Lau and L.-M. Liu, J. Phys. Chem. Lett., 2015, 6, 5002-5008.

44. L. Kou, T. Frauenheim and C. Chen, J. Phys. Chem. Lett., 2014, 5, 2675-2681.

45. V. Kumar, J. R. Brent, M. Shorie, H. Kaur, G Chadha, A. G. Thomas, E. A. Lewis, A. P. Rooney, L. Nguyen, X. L. Zhong, M. G. Burke, S. J. Haigh, A. Walton, P. D. McNaughter, A. A. Tedstone, N. Savjani, C. A. Muryn, P. O'Brien, A. K. Ganguli, D. J. Lewis and P. Sabherwal, ACS Appl. Mater. Interfaces, 2016, 8, 22860-22868.

46. S. Konabe, S. Kawabata and T. Yamamoto, Surf Interface Anal., 2016, 48, 1231-1234.

47. R. Fei, A. Faghaninia, R. Soklaski, J.-A. Yan, C. Lo and L. Yang, Nano Lett., 2014, 14, 6393-6399.

48. J. Dai and X. C. Zeng, RSC Adv., 2014, 4, 4801748021.

49. D. Hanlon, C. Backes, E. Doherty, C. S. Cucinotta, N. C. Berner, C. Boland, K. Lee, A. Harvey, P. Lynch, Z. Gholamvand, S. Zhang, K. Wang, G. Moynihan, A. Pokle, Q. M. Ramasse, N. McEvoy, W. J. Blau, J. Wang, G. Abellan, F. Hauke, A. Hirsch, S. Sanvito, D. D. O’Regan, G. S. Duesberg, V. Nicolosi and J. N. Coleman, Nat Commun, 2015, 6, 8563.

50. J. R. Brent, A. K. Ganguli, V. Kumar, D. J. Lewis, P. D. McNaughter, P. O'Brien, P. Sabherwal and A. A. Tedstone, RSC Adv., 2016, 6, 86955-86958.

51. K. S. Novoselov, A. K. Geim, S. V. Morozov, D. Jiang, M. I. Katsnelson, I. V. Grigorieva, S. V. Dubonos and A. A. Firsov, Nature, 2005, 438, 197-200.

52. K. S. Novoselov, D. Jiang, F. Schedin, T. J. Booth, V. V. Khotkevich, S. V. Morozov and A. K. Geim, Proc. Natl. Acad. Sci. U. S. A., 2005, 102, 10451-10453.

53. R. V. Gorbachev, I. Riaz, R. R. Nair, R. Jalil, L. Britnell, B. D. Belle, E. W. Hill, K. S. Novoselov, K. Watanabe, T. Taniguchi, A. K. Geim and P. Blake, Small, 2011, 7, 465-468.

54. Z. Yin, H. Li, H. Li, L. Jiang, Y. Shi, Y. Sun, G. Lu, Q. Zhang, X. Chen and H. Zhang, ACS Nano, 2012, 6, 74-80. Gan, J. Wu, H. Zhang, S. Y. Quek, M. S. Dresselhaus and Q. Xiong, Nano Lett., 2013, 13, 1007-1015.

56. H. Li, G. Lu, Y. Wang, Z. Yin, C. Cong, Q. He, L. Wang, F. Ding, T. Yu and H. Zhang, Small, 2013, 9, 1974-1981.

57. H. Fang, S. Chuang, T. C. Chang, K. Takei, T. Takahashi and A. Javey, Nano Lett., 2012, 12, 37883792.

58. Y. Cao, A. Mishchenko, G. L. Yu, E. Khestanova, A. P. Rooney, E. Prestat, A. V. Kretinin, P. Blake, M. B. Shalom, C. Woods, J. Chapman, G. Balakrishnan, I. V. Grigorieva, K. S. Novoselov, B. A. Piot, M. Potemski, K. Watanabe, T. Taniguchi, S. J. Haigh, A. K. Geim and R. V. Gorbachev, Nano Lett., 2015, 15, 4914-4921.

59. D. J. Late, B. Liu, J. Luo, A. Yan, H. S. S. R. Matte, M. Grayson, C. N. R. Rao and V. P. Dravid, Adv. Mater., 2012, 24, 3549-3554.

60. D. Teweldebrhan, V. Goyal and A. A. Balandin, Nano Lett., 2010, 10, 1209-1218.

61. H. Li, J. Wu, Z. Yin and H. Zhang, Acc. Chem. Res., 2014, 47, 1067-1075.

62. R. F. Frindt, J. Appl. Phys., 1966, 37, 1928-1929.

63. C.-G. Andres, B. Michele, M. Rianda, S. Vibhor, J. Laurens, S. J. v. d. Z. Herre and A. S. Gary, 2D Mater., 2014, 1, 011002.

64. C.-G. Andres, V. Leonardo, P. Elsa, O. I. Joshua, K. L. Narasimha-Acharya, I. B. Sofya, J. G. Dirk, B. Michele, A. S. Gary, J. V. Alvarez, W. Z. Henny, J. J. Palacios and S. J. v. d. Z. Herre, 2D Mater., 2014, 1, 025001.

65. A. Reina, H. Son, L. Jiao, B. Fan, M. S. Dresselhaus, Z. Liu and J. Kong, J. Phys. Chem. C, 2008, 112, 1774117744.

66. J. Kang, D. Shin, S. Bae and B. H. Hong, Nanoscale Horiz., 2012, 4, 5527-5537.

67. H. Li, J. Wu, X. Huang, Z. Yin, J. Liu and H. Zhang, ACS Nano, 2014, 8, 6563-6570.

68. P. Blake, E. W. Hill, A. H. C. Neto, K. S. Novoselov, D. Jiang, R. Yang, T. J. Booth and A. K. Geim, App. Phys. Lett., 2007, 91.

69. M. Batmunkh, M. Bat-Erdene and J. G. Shapter, Adv. Mater., 2016, n/a-n/a.

70. Y. Hernandez, V. Nicolosi, M. Lotya, F. M. Blighe, Z. Sun, S. De, I. T. McGovern, B. Holland, M. Byrne, Y. K. Gun'ko, J. J. Boland, P. Niraj, G. Duesberg, S. Krishnamurthy, R. Goodhue, J. Hutchison, V. Scardaci, A. C. Ferrari and J. N. Coleman, Nat. Nanotechnol., 2008, 3, 563-568.

71. E. Varrla, C. Backes, K. R. Paton, A. Harvey, Z. Gholamvand, J. McCauley and J. N. Coleman, Chem. Mater., 2015, 27, 1129-1139.

72. L. Niu, J. N. Coleman, H. Zhang, H. Shin, M. Chhowalla and Z. Zheng, Small, 2016, 12, 272-293.

73. J. N. Coleman, M. Lotya, A. O'Neill, S. D. Bergin, P. J. King, U. Khan, K. Young, A. Gaucher, S. De, R. J. Smith, I. V. Shvets, S. K. Arora, G. Stanton, H.-Y. Kim, K. Lee, G. T. Kim, G. S. Duesberg, T. Hallam, J. J. Boland, J. J. Wang, J. F. Donegan, J. C. Grunlan, G. Moriarty, A. Shmeliov, R. J. Nicholls, J. M. Perkins, E. M. Grieveson, K. Theuwissen, D. W. McComb, P. D. Nellist and V. Nicolosi, Science, 2011, 331, 568-571. 
74. J. R. Brent, D. J. Lewis, T. Lorenz, E. A. Lewis, N. Savjani, S. J. Haigh, G. Seifert, B. Derby and P. O'Brien, J. Am. Chem. Soc., 2015, 137, 12689-12696. 75. Y. Huafeng, W. Freddie, G. Elias, L. Edward, B. 93 Liam, F. Alexandre, P. Vincenzo, H. Sarah, B. David and C. Cinzia, 2D Mater., 2014, 1, 011012. 76. A. A. Tedstone, D. J. Lewis and P. O'Brien, Chem. Mater., 2016, 28, 1965-1974.

77. D. J. Lewis, A. A. Tedstone, X. L. Zhong, E. A. Lewis, A. Rooney, N. Savjani, J. R. Brent, S. J. Haigh, M. G. Burke, C. A. Muryn, J. M. Raftery, C. Warrens, K. West, S. Gaemers and P. O'Brien, Chem. Mater., 2015, 27, 1367-1374.

78. N. Al-Dulaimi, E. A. Lewis, D. J. Lewis, S. K. Howell, S. J. Haigh and P. O'Brien, Chem. Commun., 2016, 52, 7878-7881.

79. F. Withers, H. Yang, L. Britnell, A. P. Rooney, E. Lewis, A. Felten, C. R. Woods, V. Sanchez Romaguera, T. Georgiou, A. Eckmann, Y. J. Kim, S. G. Yeates, S. J. Haigh, A. K. Geim, K. S. Novoselov and C. Casiraghi, Nano Lett., 2014, 14, 3987-3992. 80. T. J. Mason and J. P. Lorimer, in Appl. Sonochemistry, Wiley-VCH Verlag GmbH \& Co. KGaA, Editon edn., 2003, pp. 25-74. 81. J. R. Brent, N. Savjani, E. Lewis, S. Haigh, D. Lewis and P. O'Brien, Chem. Commun., 2014, 50, 1333813341.

82. J. Kang, J. D. Wood, S. A. Wells, J.-H. Lee, X. Liu, K.-S. Chen and M. C. Hersam, ACS Nano, 2015, 9, 35963604.

83. C. C. Nascentes, M. Korn, C. S. Sousa and M. A. Z. Arruda, J. Braz. Chem. Soc., 2001, 12, 57-63.

84. H. M. Santos and J. L. Capelo, Talanta, 2007, 73, 795-802.

85. C. Backes, T. M. Higgins, A. Kelly, C. Boland, A. Harvey, D. Hanlon and J. N. Coleman, Chem. Mater. 2016.

86. P. Yasaei, B. Kumar, T. Foroozan, C. Wang, M. Asadi, D. Tuschel, J. E. Indacochea, R. F. Klie and A. Salehi-Khojin, Adv. Mater., 2015, 27, 1887-1892.

87. A. H. Woomer, T. W. Farnsworth, J. Hu, R. A. Wells, C. L. Donley and S. C. Warren, ACS Nano, 2015, 9, 8869-8884.

88. Y. Zhang, X. Rui, Y. Tang, Y. Liu, J. Wei, S. Chen, W. R. Leow, W. Li, Y. Liu, J. Deng, B. Ma, Q. Yan and X. Chen, Adv. Energy Mater., 2016, 6, 1502409-n/a.

89. W. Zhao, Z. Xue, J. Wang, J. Jiang, X. Zhao and T. Mu, ACS Appl. Mater. Interfaces, 2015, 7, 27608-27612. 90. C. Backes, R. J. Smith, N. McEvoy, N. C. Berner, D. McCloskey, H. C. Nerl, A. O'Neill, P. J. King, T. Higgins, D. Hanlon, N. Scheuschner, J. Maultzsch, L. Houben, G. S. Duesberg, J. F. Donegan, V. Nicolosi and J. N Coleman, Nat Commun, 2014, 5, 4576.

91. C. Backes, B. M. Szydłowska, A. Harvey, S. Yuan, V. Vega-Mayoral, B. R. Davies, P.-I. Zhao, D. Hanlon, E. J. G. Santos, M. I. Katsnelson, W. J. Blau, C. Gadermaier and J. N. Coleman, ACS Nano, 2016, 10, 1589-1601.

92. C. Zhu, F. Xu, L. Zhang, M. Li, J. Chen, S. Xu, G. Huang, W. Chen and L. Sun, Chem. Eur. J., 2016, 22, 7357-7362.

93. Z. Sun, H. Xie, S. Tang, X.-F. Yu, Z. Guo, J. Shao, H. Zhang, H. Huang, H. Wang and P. K. Chu, Angew. Chem. Int. Ed., 2015, 127, 11688-11692.

94. L.-F. Gao, J.-Y. Xu, Z.-Y. Zhu, C.-X. Hu, L. Zhang, Q. Wang and H.-L. Zhang, Nanoscale Horiz., 2016, 8, 15132-15136.

95. M. Serrano-Ruiz, M. Caporali, A. Ienco, V. Piazza, S. Heun and M. Peruzzini, Adv. Mater. Interfaces, 2016, 3, 1500441-n/a.

96. Z. Guo, H. Zhang, S. Lu, Z. Wang, S. Tang, J. Shao, Z. Sun, H. Xie, H. Wang, X.-F. Yu and P. K. Chu, Adv. Funct. Mater., 2015, 25, 6996-7002.

97. J.-Y. Xu, L.-F. Gao, C.-X. Hu, Z.-Y. Zhu, M. Zhao, Q. Wang and H.-L. Zhang, Chem. Commun., 2016, 52, 8107-8110.

98. D. Li, A. E. Del Rio Castillo, H. Jussila, G. Ye, Z. Ren, J. Bai, X. Chen, H. Lipsanen, Z. Sun and F. Bonaccorso, Appl. Mater. Today, 2016, 4, 17-23.

99. D. J. Late, Micropor. Mesopor. Mater., 2016, 225, 494-503.

100. S. Seo, H. U. Lee, S. C. Lee, Y. Kim, H. Kim, J. Bang, J. Won, Y. Kim, B. Park and J. Lee, Sci. Rep., 2016, 6, 23736.

101. P. Yasaei, A. Behranginia, T. Foroozan, M. Asadi, K. Kim, F. Khalili-Araghi and A. Salehi-Khojin, ACS Nano, 2015, 9, 9898-9905.

102. Y. Hernandez, M. Lotya, D. Rickard, S. D. Bergin and J. N. Coleman, Langmuir, 2009, 26, 3208-3213.

103. Y. Jing, X. Zhang and Z. Zhou, WIREs Comput. Mol. Sci., 2016, 6, 5-19.

104. V. Sresht, A. A. H. Pádua and D. Blankschtein, ACS Nano, 2015, 9, 8255-8268.

105. M. B. Dines, Mater. Res. Bull., 1975, 10, 287-291. 106. M. S. Dresselhaus and G. Dresselhaus, Adv. Phys., 2002, 51, 1-186.

107. E. Benavente, M. A. Santa Ana, F. Mendizabal and G. Gonzalez, Coord. Chem. Rev., 2002, 224, 87-109.

108. M. Chhowalla, H. S. Shin, G. Eda, L. J. Li, K. P. Loh and H. Zhang, Nat. Chem., 2013, 5, 263-275.

109. H. Matte, A. Gomathi, A. K. Manna, D. J. Late, R. Datta, S. K. Pati and C. N. R. Rao, Angew. Chem. Int. Ed., 2010, 49, 4059-4062.

110. G. Eda, H. Yamaguchi, D. Voiry, T. Fujita, M. W. Chen and M. Chhowalla, Nano Lett., 2011, 11, 51115116.

111. C.-J. Shih, A. Vijayaraghavan, R. Krishnan, R. Sharma, J.-H. Han, M.-H. Ham, Z. Jin, S. Lin, G. L. C. Paulus, N. F. Reuel, Q. H. Wang, D. Blankschtein and M. S. Strano, Nat. Nano., 2011, 6, 439-445.

112. T. Nishi, Y. Maruyama, T. Inabe and I. Shirotani, Synth. Met., 1987, 18, 559-564.

113. M. C. Stan, J. von Zamory, S. Passerini, T. Nilges and M. Winter, J. Mater. Chem. A, 2013, 1, 5293-5300. 
114 114. M. Lotya, Y. Hernandez, P. J. King, R. J. Smith, V. Nicolosi, L. S. Karlsson, F. M. Blighe, S. De, Z. Wang, I. T. McGovern, G. S. Duesberg and J. N. Coleman, Journal of the American Chemical Society, 2009, 131, 36113620 .

115 115. L. Chen, G. Zhou, Z. Liu, X. Ma, J. Chen, Z. Zhang, X. Ma, F. Li, H.-M. Cheng and W. Ren, Adv. Mater., 2016, 28, 510-517.

116 116. H. Wang, X. Yang, W. Shao, S. Chen, J. Xie, X. Zhang, J. Wang and Y. Xie, J. Am. Chem. Soc., 2015, 137, 11376-11382.

117 117. Y. Zhao, H. Wang, H. Huang, Q. Xiao, Y. Xu, Z. Guo, H. Xie, J. Shao, Z. Sun, W. Han, X.-F. Yu, P. Li and P. K. Chu, Angew. Chem. Int. Ed., 2016, 128, 5087-5091.

118 118. C. Sun, L. Wen, J. Zeng, Y. Wang, Q. Sun, L. Deng, C. Zhao and Z. Li, Biomaterials, 2016, 91, 81-89.

119 119. H. S. Choi, W. Liu, F. Liu, K. Nasr, P. Misra, M. G. Bawendi and J. V. Frangioni, Nat. Nanotechnol., 2010, 5, 42-47.

120 120. H. Soo Choi, W. Liu, P. Misra, E. Tanaka, J. P. Zimmer, B. Itty Ipe, M. G. Bawendi and J. V. Frangioni, Nat. Biotech., 2007, 25, 1165-1170.

121 121. M. Lotya, Y. Hernandez, P. J. King, R. J. Smith, V. Nicolosi, L. S. Karlsson, F. M. Blighe, S. De, Z. Wang, I. T. McGovern, G. S. Duesberg and J. N. Coleman, J. Am. Chem. Soc., 2009, 131, 3611-3620.

122 122. R. J. Smith, P. J. King, M. Lotya, C. Wirtz, U. Khan, S. De, A. O'Neill, G. S. Duesberg, J. C. Grunlan, G. Moriarty, J. Chen, J. Wang, A. I. Minett, V. Nicolosi and J. N. Coleman, Adv. Mater., 2011, 23, 3944-3948.

123. J. Kang, S. A. Wells, J. D. Wood, J.-H. Lee, X. Liu, C. R. Ryder, J. Zhu, J. R. Guest, C. A. Husko and M. C. Hersam, Proc. Natl. Acad. Sci. U. S. A., 2016.

124. K. R. Paton, E. Varrla, C. Backes, R. J. Smith, U. Khan, A. O'Neill, C. Boland, M. Lotya, O. M. Istrate, P. King, T. Higgins, S. Barwich, P. May, P. Puczkarski, I. Ahmed, M. Moebius, H. Pettersson, E. Long, J. Coelho, S. E. O'Brien, E. K. McGuire, B. M. Sanchez, G. S. Duesberg, N. McEvoy, T. J. Pennycook, C. Downing, A. Crossley, V. Nicolosi and J. N. Coleman, Nat Mater, 2014, 13, 624-630.

125. E. Varrla, K. R. Paton, C. Backes, A. Harvey, R. J. Smith, J. McCauley and J. N. Coleman, Nanoscale Horiz., 2014, 6, 11810-11819.

126 126. X. Feng, G. Binghui, C. Jing, N. Arokia, L. X. Linhuo, M. Hongyu, M. Huihua, Z. Chongyang, X. Weiwei, L. Zhengrui, L. Shengli, Y. Kaihao, W. Lijun, C. Yiping, S. Litao and Z. Yimei, 2D Mater., 2016, 3, 025005.

127 127. K.-G. Zhou, M. Zhao, M.-J. Chang, Q. Wang, X.-Z. Wu, Y. Song and H.-L. Zhang, Small, 2015, 11, 694-701. 128. U. Khan, A. O'Neill, H. Porwal, P. May, K. Nawaz and J. N. Coleman, Carbon, 2012, 50, 470-475.

129 129. G. Eda, G. Fanchini and M. Chhowalla, Nat. Nanotechnol., 2008, 3, 270-274.

130 130. H. Chen, M. B. Müller, K. J. Gilmore, G. G. Wallace and D. Li, Adv. Mater., 2008, 20, 3557-3561.
131. D. Li, M. B. Muller, S. Gilje, R. B. Kaner and G. G. Wallace, Nat. Nanotechnol., 2008, 3, 101-105.

132. Y. Zhang, H. Wang, Z. Luo, H. T. Tan, B. Li, S. Sun, Z. Li, Y. Zong, Z. J. Xu, Y. Yang, K. A. Khor and Q. Yan, Adv. Energy Mater., 2016, 6, n/a-n/a.

133. H. Kaur, S. Yadav, A. Srivastava, N. Singh, J. J. Schneider, O. Sinha, V. V. Agrawal and R. Srivastava, arXiv, 2016, arXiv:1409.0459.

134. S. Stankovich, D. A. Dikin, G. H. B. Dommett, K. M. Kohlhaas, E. J. Zimney, E. A. Stach, R. D. Piner, S. T. Nguyen and R. S. Ruoff, Nature, 2006, 442, 282-286.

135. C. Zhi, Y. Bando, C. Tang, H. Kuwahara and D. Golberg, Adv. Mater., 2009, 21, 2889-2893.

136. F. Torrisi, T. Hasan, W. Wu, Z. Sun, A. Lombardo, T. S. Kulmala, G.-W. Hsieh, S. Jung, F. Bonaccorso, P. J. Paul, D. Chu and A. C. Ferrari, ACS Nano, 2012, 6, 29923006.

137. W. Luo, R. Yang, J. Liu, W. Zhu and G. Xia, arXiv preprint arXiv:1601.04103, 2016.

138. M. B. Erande, S. R. Suryawanshi, M. A. More and D. J. Late, Eur. J. Inorg. Chem., 2015, 2015, 3102-3107.

139. C. Schliehe, B. H. Juarez, M. Pelletier, S. Jander, D. Greshnykh, M. Nagel, A. Meyer, S. Foerster, A. Kornowski, C. Klinke and H. Weller, Science, 2010, 329, 550-553.

140. N. Savjani, E. A. Lewis, M. A. Bissett, J. R. Brent, R. A. W. Dryfe, S. J. Haigh and P. O'Brien, Chem. Mater., 2016, 28, 657-664.

141. C. Altavilla, M. Sarno and P. Ciambelli, Chem. Mater., 2011, 23, 3879-3885.

142. Z. Yang, J. Hao, S. Yuan, S. Lin, H. M. Yau, J. Dai and S. P. Lau, Adv. Mater., 2015, 27, 3748-3754.

143. X. Ling, H. Wang, S. Huang, F. Xia and M. S. Dresselhaus, Proc. Natl. Acad. Sci. U. S. A., 2015, 112, 4523-4530.

144. A. Carvalho and A. H. C. Neto, ACS Cent. Sci., 2015, 1, 289-291.

145. J. D. Wood, S. A. Wells, D. Jariwala, K.-S. Chen, E. Cho, V. K. Sangwan, X. Liu, L. J. Lauhon, T. J. Marks and M. C. Hersam, Nano Lett., 2014, 14, 6964-6970.

146. J. O. Island, G. A. Steele, H. S. J. v. d. Zant and A. Castellanos-Gomez, 2D Mater., 2015, 2, 011002.

147. Y. Huang, J. Qiao, K. He, S. Bliznakov, E. Sutter, X. Chen, D. Luo, F. Meng, D. Su, J. Decker, W. Ji, R. S. Ruoff and P. Sutter, Chem. Mater., 2016.

148. A. Favron, E. Gaufrès, F. Fossard, A.-L. PhaneufL'Heureux, N. Y. W. Tang, P. L. Lévesque, A. Loiseau, R. Leonelli, S. Francoeur and R. Martel, Nat Mater, 2015, 14, 826-832.

149. A. Castellanos-Gomez, L. Vicarelli, E. Prada, J. O. Island, K. L. Narasimha-Acharya, S. I. Blanter, D. J. Groenendijk, M. Buscema, G. A. Steele, J. V. Alvarez, H. W. Zandbergen, J. J. Palacios and H. S. J. v. d. Zant, 2D Mater., 2014, 1, 025001.

150. Y. Cai, Q. Ke, G. Zhang and Y.-W. Zhang, J Phys. Chem. C, 2015, 119, 3102-3110. 
151 151. G. C. Constantinescu and N. D. M. Hine, Nano Lett., 2016, 16, 2586-2594.

152 152. Y. Stehle, H. M. Meyer, R. R. Unocic, M. Kidder, G. Polizos, P. G. Datskos, R. Jackson, S. N. Smirnov and I. V. Vlassiouk, Chem. Mater., 2015, 27, 8041-8047.

153 153. Q. Wu, W. Wongwiriyapan, J.-H. Park, S. Park, S. J. Jung, T. Jeong, S. Lee, Y. H. Lee and Y. J. Song, Current Applied Physics, 2016, 16, 1175-1191.

154 154. M. T. Edmonds, A. Tadich, A. Carvalho, A. Ziletti, K. M. O'Donnell, S. P. Koenig, D. F. Coker, B. Özyilmaz, A. H. C. Neto and M. S. Fuhrer, ACS Appl. Mater. Interfaces, 2015, 7, 14557-14562.

155 155. J. B. Smith, D. Hagaman and H.-F. Ji, Nanotechnology, 2016, 27, 215602.

156 156. D. Geng, B. Wu, Y. Guo, L. Huang, Y. Xue, J. Chen, G. Yu, L. Jiang, W. Hu and Y. Liu, Proc. Natl. Acad. Sci. U. S. A., 2012, 109, 7992-7996.

157 157. T. Oznuluer, E. Pince, E. O. Polat, O. Balci, O. Salihoglu and C. Kocabas, App. Phys. Lett., 2011, 98, 183101.

158 158. A. Koma, J. Cryst. Growth, 1999, 201-202, 236241.

159 159. C. Tan and H. Zhang, J. Am. Chem. Soc., 2015, 137, 12162-12174.

160 160. L. C. Gomes and A. Carvalho, Phys. Rev. B, 2015, 92, 085406 .

161 161. M. Baba, F. Izumida, Y. Takeda and A. Morita, Jpn. J. Appl. Phys., 1989, 28, 1019-1022.

162 162. H. Krebs, H. Weitz and K. H. Worms, Z. anorg. allg. Chem., 1955, 280, 119-133.

163 163. L.-D. Zhao, S.-H. Lo, Y. Zhang, H. Sun, G. Tan, C. Uher, C. Wolverton, V. P. Dravid and M. G. Kanatzidis, Nature, 2014, 508, 373-377.

164 164. H. Y. Lv, W. J. Lu, D. F. Shao and Y. P. Sun, Phys. Rev. B, 2014, 90, 085433.

165 165. Y. Deng, Z. Luo, N. J. Conrad, H. Liu, Y. Gong, S. Najmaei, P. M. Ajayan, J. Lou, X. Xu and P. D. Ye, ACS Nano, 2014, 8, 8292-8299.

166 166. J. Dai and X. C. Zeng, J. Phys. Chem. Lett., 2014, 5, 1289-1293.

167 167. K. Manabendra, K. Chandan, C. Biswanath, N. G. Satyendra, H. N. Mit, J. Manish, A. K. Sood and D. Anindya, Nanotechnology, 2015, 26, 485704.

168 168. M. A. Huber, F. Mooshammer, M. Plankl, L. Viti, F. Sandner, L. Z. Kastner, T. Frank, J. Fabian, M. S. Vitiello, T. L. Cocker and R. Huber, Nat. Nano., 2016, DOI: 10.1038/nnano.2016.1261.

169 169. D. J. Lewis, V. Dore, N. J. Rogers, T. K. Mole, G. B. Nash, P. Angeli and Z. Pikramenou, Langmuir, 2013, 29, 14701-14708. 